\title{
AUTOMATIC CONTROL OF AN ANTI-TANK GUIDED MISSILE BASED ON POLYNOMIAL FUNCTIONS
}

\author{
ZBigniew Koruba, Łukasz Nocoń \\ Kielce University of Technology, Faculty of Mechatronics and Machine Design, Kielce, Poland \\ e-mail:ksmzko@tu.kielce.pl; waldek.afro@op.pl
}

\begin{abstract}
The paper presents algorithms of automatic control of an intelligent anti-tank guided missile with the possibility of attacking a target from the upper ceiling (top attack). Polynomial functions have been used to determine program trajectories. The numerical analysis of chosen algorithms has been performed. The results of work are presented in a graphical form. It appears from the conducted research that the proposed algorithms of automatic control of the anti-tank guided missile work correctly during attack from the upper ceiling, both movable and fixed.
\end{abstract}

Keywords: automatic control, anti-tank guided missile, dynamics, control

\section{Nomenclature}

\begin{tabular}{|c|c|}
\hline A & - vector resultant of aerodynamic forces \\
\hline$C_{1}, C_{2}, C_{3}$ & - coefficients of aerodynamic moments (constant values adopted) \\
\hline$c_{x}, c_{y}, c_{z}$ & coefficients of aerodynamic forces (constant values adopted) \\
\hline G & gravitational acceleration \\
\hline$J_{k}, J_{o k}$ & $\begin{array}{l}\text { - main central moments of inertia of missile in relation to vertical and horizontal } \\
\text { axis of missile }\end{array}$ \\
\hline$L, m$ & length and mass of missile \\
\hline M & sum of moments of forces influencing missile \\
\hline$M_{\eta}, M_{\zeta}$ & moments of external forces in relation to missile centre of mass \\
\hline $\mathbf{P}$ & total missile thrust \\
\hline $\mathrm{Q}$ & controlling force \\
\hline$Q_{y}, Q_{z}$ & (external) forces controlling missile flight \\
\hline$r$ & - line-of-sight vector from ATGM to target \\
\hline$S$ & typical surface (area of biggest missile cross-section) \\
\hline Sxyz & system of coordinates connected with missile \\
\hline$S x_{g} y_{g} z_{g}$ & system of coordinates with respect to beginning of missile \\
\hline$S x_{v} y_{v} z_{v}$ & system of coordinates connected with flow \\
\hline$u$ & arm (distance of missile centre of mass to application of controlling force) \\
\hline $\mathbf{V}_{p}$ & velocity vector \\
\hline$Y_{k}, Z_{k}$ & disrupting forces \\
\hline$\alpha, \beta$ & - missile angle-of-attack and sideslip angle \\
\hline$\gamma, \chi$ & $\begin{array}{l}\text { - flight-path angle in vertical plane and horizontal plane (inclination and dec- } \\
\text { lination angles of missile velocity vector) }\end{array}$ \\
\hline$\varepsilon$ & line-of-sight angle \\
\hline$\vartheta, \psi$ & pitch and yaw angle \\
\hline$\lambda_{x}, \lambda_{y}$ & - coefficients of aerodynamic forces \\
\hline & - air density for normal conditions \\
\hline
\end{tabular}




\section{Introduction}

In this paper, an Anti-Tank Guided Missile (ATGM) of the third generation is discussed, which is marked by attacking a target by passive guidance, limiting possibilities of disruptions of its flight, and the ability to choose a target during its flight. ATGM, due to their guidance onto a target, can be divided into three basic groups:

a) "fire and forget" missile - fully automatic flight to a target,

b) "fire-observe-correct" missile - sending the image from the battlefield to the command post via a fibre-optic cable;

c) missile with an alternative guidance system - depending on the situation on the battlefield (system a or b).

The missile from the second group is equipped with an IIR (Imaging Infra Red) head with which it is possible to watch the battlefield, distinguish the target, send that image to the monitor on a shooting position via the fibre-optic cable unwound during flight. The shooter can correct the path of the missile during its flight by sending corrective signals to missile performing systems, with the use of the fibre-optic cable, till reaching the target or the point from which the flight control switches to self-guidance. It needs to be emphasized that the missile is equipped in that case with a head with stabilized TV and/or IIR camera and during the flight it sends the picture "seen" by the camera to the control station through the fibre-optic cable. The operator, seeing the picture of the battlefield, chooses the target and with the use of the fibre-optic cable sends the signals controlling the missile flight. That control is manual. The whole missile guiding process is carried out from the control station with simultaneous analysis of the picture in real time.

The system with "a man in the control loop" gives many possibilities when carrying out an attack on an armoured vehicle, ship or building. The presence of the operator allows one to use a simple, resistant to disruptions (fibre-optic cable), and also effective system of attack. The system allows for fighting the targets unseen from the control station, attacking the target located behind a natural or artificial obstacle from the upper ceiling, and even some air (slow moving) targets. An approximate location of the target is sufficient for firing the missile. The missile range is limited only by the length of the fibre-optic cable, though the application of self-guidance to the target in the last stage of flight can increase that range. We say that controlling the missiles through the fibre-optic cable is done on the basis of "fire-observe-correct" principle. It allows not only for precise guiding to the target, but also for changing the target during flight. The drawback of the discussed guidance systems are operator's manual limitations demanding a relatively small velocity of missile flight, hence the possibility of destroying it with anti-rocket missiles.

The third generation ATGM can attack a target from the upper ceiling or directly along the line of aiming. The missile flight is not controlled from the shooting point (launcher) as it was in older generation missiles. In the case of decision about attack from the upper ceiling, after mounting a missile to the launcher, the shooter sets a "marker" visible in the optical system over the chosen target and according to that adjusts the missile on the launcher.

Two models of target attack are possible: the attack along the line-of-sight (LOS) and the attack from the upper half-zone (ceiling) (Koruba and Nocon, 2012). In the first case, the target is attacked from horizontal flight, and in the second case from diving flight, which allows hitting the target in weakly armoured places.

In the case of decision to attack from the upper ceiling, the missile is guided to the target independently (according to the programmed algorithm and the intended length of the target from the launcher) on such a path that the passive IIR head intercepts the target. From that moment, the missile carries out self-guidance on the target. 
In the case of decision about a direct attack, the missile is lead to the target by a program on a flat path (close to a straight line). If, when approaching the target, the IIR head area of vision is narrowed so much that the target is intercepted, the target self-guidance is carried out.

Due to the short-range, the missile flies almost in the straight line. It can be aimed in such a way that it flew over the target (top attack), as well as hit straight in the target (direct attack). Cumulative Explosively Formed Projectile (EFP) in the battle head enables the attack on the active armour of the target. The launcher is equipped with a day-night sight. An auto-pilot assures the desired path of flight. Many missiles have cumulative heads of the "tandem" type and a precise preliminary self-guidance and self-guidance in the final part of the flight (optical target seeker and detector sensitive to weak thermal contrast).

A big advantage of the discussed missile is the possibility of conducting its start and directing its flight from the launcher located behind the hiding place. It is possible thanks to the fact that after vertical start (e.g. at an angle of $45^{\circ}$ ) in the initial flight section, the missile motions according to the set program with the use of inert measurement unit cooperating with the GPS system. At a consecutive flight stage, the missile is guided by an operator. Usually in such a case, a TV and thermal imaging camera is mounted on a special gyroscope-stabilized platform in two planes thanks to which high stability and sharpness of the transmitted picture is obtained. Moreover, high sensitivity and other characteristics of the detectors used in the head ensure elimination of the background thanks to which the missile of that kind can be effective on the battlefield in adverse weather conditions during the day and night indicating high resistance to thermal interferences.

The most significant drawbacks of controlling the missile through the fibre-optic cable include:

- limitations of range resulting from the length of the fibre-optic cable,

- limitations of missile velocity (with speeds greater than $200 \mathrm{~m} / \mathrm{s}$ the fibre-optic cable unrolling from a reel breaks),

- limitations of the rate of fire resulting from longer time to target,

- high operating costs due to the need of servicing of fibre-optic reels and tracking heads,

- necessity of constant seeing of the target (disappearance of the visibility of the target above a couple of seconds makes it impossible to guide).

In connection with the above, for a long-range ATGM with an increased range and high velocity of flight, with the ability of individual searching and identification of objects - one should look for some other alternative solutions.

The paper proposes algorithms allowing for fulfilling the above requirements. It needs to be emphasized that automatic control of ATGM with the use of polynomial functions influences the minimization of human interference in the process of guiding third generation ATGM's from the moment of firing till destruction of the target ("fire and forget", and even "set and forget").

\section{The control algorithm of an anti-tank guided missile}

\subsection{The concept of the control algorithm of an anti-tank guided missile}

The fulfilment of the algorithm consists in controlling the flight in such a way that it follows the determined path of flight. In this case, follows the polynomial curve. The most frequently used method of control includes various variants of error control. This method consists in introducing controlling forces $Q$ dependent on the current deviation (error) of the carried out flight from the set flight (Evans, 1990). 
In order to determine the error, it is necessary to introduce feedback informing the control system about the parameters of the flight carried out as a result of the control. The methods of obtaining that information differ in various solutions, but they can be divided into two groups (Koruba, 2008).

Autonomous systems independently determine their location, whether on the basis of sensors tracking the parameters of flight (such as velocity and direction), or applying other navigation methods, e.g. satellite navigation GPS (first section of flight is presented in Fig. 1).

Non-autonomous systems using Observation and Tracking Devices (OTD) for obtaining the required information (self-guidance systems - the second section of flight shown in Fig. 1) or control signals, i.e. "commands" (remote self-guidance systems). OTD can also direct a laser beam into which the missile is fired (or to the reflection of which the missile is guided); the control system maintains the missile in the said beam (Fig. 1).

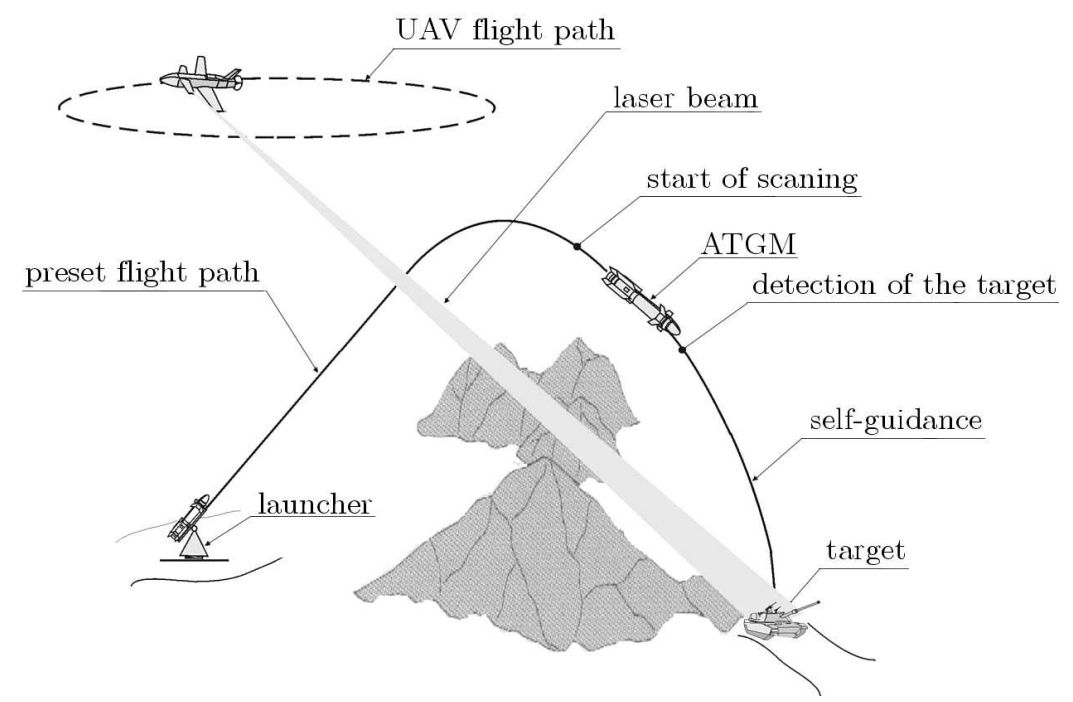

Fig. 1. General view of the guidance of third generation ATGM with the use of a laser beam emitted from the Unmanned Aerial Vehicle

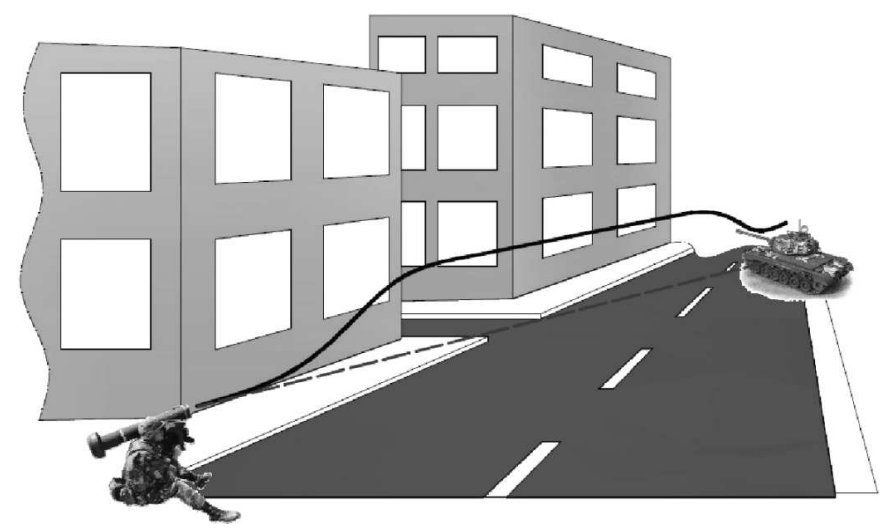

Fig. 2. General view of the self-guidance of third generation close-range ATGM

The considerations on the algorithm include two possibilities of attack on armoured objects: slowly moving targets more than a dozen or twenty kilometres away (Fig. 1) requiring long-range missiles the flight of which is divided into programmed flight, tracking and self-guidance, as well as targets in the immediate range of vision (Fig. 2). In both cases, the algorithm assumes attack on the upper surface of the armoured object. In the first case, the attack takes place from diving flight (Yanushevsky, 2008). The drawback of that solution is the limited possibility of 
manoeuvring following the moving target at the final stage of attack when the flight trajectory of ATGM assumes almost vertical form. In the second case, the attack is carried out along the direction of the Line-Of-Sight (LOS) with the ceiling during the programmed flight set at approx. 4 meters above the target (uplift of flight trajectory) and the decrease of the trajectory in the phase of attack in such a way that the ATGM flies just above the target.

\subsection{The equations of motion of the anti-tank guided missile}

Assuming that the missile is a rigid solid, its motions in dense layers of atmosphere (close to the Earth's surface), the equations of its motion in function of time are as follows (Koruba and Osiecki, 2006; Siouris, 2004)

$$
\begin{aligned}
& \frac{d V_{P}}{d t}=\frac{P}{m} \cos \alpha \cos \beta-g \sin \gamma \cos \chi-\lambda_{x} V_{P}^{2} \\
& \frac{d \gamma}{d t}=\left(\frac{P}{m} \sin \alpha \cos \beta-g \cos \gamma+\lambda_{y} V_{P}^{2} \alpha+\frac{Q_{y}+Y_{k}}{m}\right) \frac{1}{V_{P} \cos \chi} \\
& \frac{d \chi}{d t}=\frac{P}{m V_{P}} \sin \beta+\frac{g}{V_{P}} \sin \gamma \sin \chi-\lambda_{z} V_{P} \beta-\frac{Q_{z}+Z_{k}}{m V_{P}}
\end{aligned}
$$

and

$$
\begin{aligned}
& \ddot{\vartheta} \cos \psi-\dot{\vartheta} \dot{\psi} \sin \psi+\left(\frac{J_{o k}}{J_{k}}-1\right) \dot{\psi} \dot{\vartheta} \sin \psi=-D_{1} \frac{V^{2}}{I} \alpha-D_{2} V \dot{\alpha}-D_{3} V \dot{\vartheta}+\frac{M_{Q \zeta}}{J_{k}} \\
& \ddot{\psi}-\left(\frac{J_{o k}}{J_{k}}-1\right) \dot{\vartheta}^{2} \sin \psi \cos \psi=-D_{1} \frac{\beta}{L} V^{2}-D_{2} V \dot{\beta}-D_{3} V \dot{\psi}+\frac{M_{Q \eta}}{J_{k}}
\end{aligned}
$$

whereas, the same equations of motion in function of variable $x$ have the form (Koruba and Osiecki, 2006)

$$
\begin{aligned}
& \frac{d V_{P}}{d x}=\frac{\sqrt{1+y^{\prime 2}(x)}}{V_{P}}\left(\frac{P}{m}-g y^{\prime}(x)-\lambda_{x} V_{P}^{2}\right) \\
& \frac{d \gamma}{d x}=\frac{\sqrt{1+y^{\prime 2}(x)}}{V_{P}}\left[\left(\frac{P}{m V_{P}}+\lambda_{y} V_{P}\right)(\vartheta-\gamma)-\frac{g}{V_{P}} \cos \gamma+\frac{Q_{y}+Y_{k}}{m V_{P}}\right] \\
& \frac{d \chi}{d x}=\frac{\sqrt{1+y^{\prime 2}(x)}}{V_{P}}\left[\left(\frac{P}{m V_{P}}+\lambda_{y} V_{P}\right)(\psi-\chi)-\frac{g}{V_{P}} \chi \cos \gamma+\frac{Q_{z}+Z_{k}}{m V_{P}}\right]
\end{aligned}
$$

and

$$
\begin{aligned}
& \frac{d^{2} \vartheta}{d x^{2}}-\left(\frac{J_{o k}}{J_{k}}-1\right) \frac{v^{2}}{1-y^{\prime 2}(x)}\left(\frac{d \vartheta}{d x}\right)^{2}=-\left(\frac{d v}{d x}-v \frac{y^{\prime}(x) y^{\prime \prime}(x)}{1+y^{\prime 2}(x)}\right) \frac{d \vartheta}{d x}-D_{1} \frac{\alpha}{L}\left[1+y^{\prime 2}(x)\right] \\
& \quad+D_{2} \frac{y^{\prime \prime}(x)}{\sqrt{1+y^{\prime 2}(x)}}-\left(D_{2}+D_{3}\right) \sqrt{1+y^{\prime 2}(x)} \frac{d \vartheta}{d x}+\frac{M_{\zeta}}{J_{k}} \frac{1}{v^{2} \cos ^{2} \gamma} \\
& \frac{d^{2} \psi}{d x^{2}}=-\left(\frac{d v}{d x}-v \frac{y^{\prime}(x) y^{\prime \prime}(x)}{1+y^{\prime 2}(x)}\right) \frac{d \psi}{d x}-D_{1} \frac{\beta}{L}\left[1+y^{\prime 2}(x)\right] \\
& \quad+D_{2} \sqrt{1+y^{\prime 2}(x)} \frac{d \chi}{d x}-\left(D_{2}+D_{3}\right) \sqrt{1+y^{\prime 2}(x)} \frac{d \psi}{d x}+\frac{M_{\eta}}{J_{k}} \frac{1}{v^{2} \cos ^{2} \gamma} \\
& \alpha=\vartheta-\gamma \quad \eta=\psi-\chi
\end{aligned}
$$

where

$$
\begin{aligned}
& D_{1}=\frac{C_{1} L}{J_{k}} \\
& D_{2}=\frac{C_{2} L}{J_{k}} \\
& D_{3}=\frac{C_{3} L}{J_{k}} \\
& \lambda_{x}=c_{x} \frac{\rho S}{2 m} \\
& \lambda_{y}=c_{y} \frac{\rho S}{2 m} \\
& \lambda_{z}=c_{z} \frac{\rho S}{2 m}
\end{aligned}
$$


Based on the determined errors between the set and carried out path, the controlling forces are calculated in the automatic pilot (auto-pilot). We assume that those forces in the simplest case can be linearly dependent on the error and on dynamics of changes of that error (controller type PD) (Koruba, 2008)

$$
\begin{array}{ll}
Q_{y}=k_{y 1} e_{y}+k_{y 2} \frac{d e_{y}}{d t} & Q_{z}=k_{z 1} e_{z}+k_{z 2} \frac{d e_{z}}{d t} \\
e_{y}(t)=\gamma^{\circ}-\gamma & e_{z}(t)=\chi^{\circ}-\chi
\end{array}
$$

where: $\gamma^{\circ}, \chi^{\circ}$ - desired angles of flight of ATGM, $k_{y 1}, k_{y 2}, k_{z 1}$ and $k_{z 2}$ - parameters of the controller.

\subsection{Numerical notation of the algorithm in vertical plane}

During the flight many factors have impact on the missile influencing its behaviour in space. Starting from aerodynamic forces, rocket engine thrust, Coriolis force, to factors disrupting its flight, as for example ordinary wind. The considerations exclude climactic conditions, wind, and other external interferences. Figure 3 presents a simplified diagram of operation of ATGM guidance (Koruba and Nocoń, 2012).

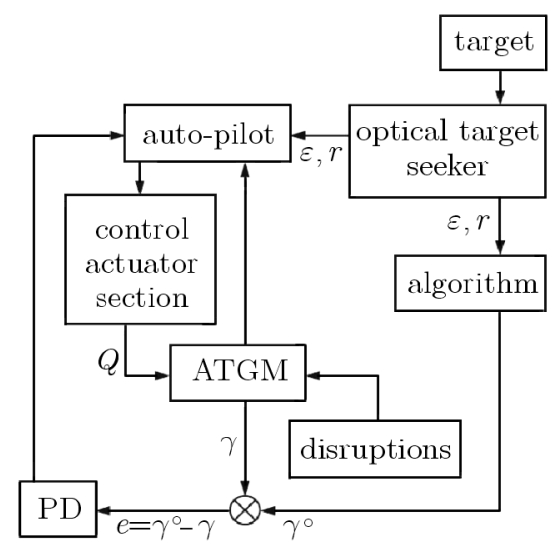

Fig. 3. Simplified diagram of ATGM guidance

The control is done by making use of the controlling force $Q$ generated by the control performance system. Control signals are calculated by the proportional and differential controller (PD). The controller argument is the deviation $e=\gamma^{\circ}-\gamma$ between the angle of tangent to the path and the desired angle of control. The angle of control $\gamma^{\circ}$ is determined on the basis of the polynomial function.

Two variants of attack are considered. In the first variant, the short-range missile is analysed attacking the target along the line-of-sight with programmed flight set approx. $4 \mathrm{~m}$ above the target (Fig. 4). In the phase of attack, ATGM moves on the polynomial curve in such a way that the missile flies above the target and attacks its upper surface (Fig. 5a). In the second case, the long-range rocket missile is considered which reaches high ceiling of the programmed flight (more than $300 \mathrm{~m}$ ) and attacks the target from diving flight. The final stage is analysed from the moment of fixing its position to the moment of hitting it (Fig. 5b).

The trajectory of flight of the anti-tank guided missile in the attack phase is described with the use of the third degree polynomial function (Grzyb and Koruba, 2011)

$$
y=a x^{3}+b x^{2}+c x+d
$$

The proper choice of coefficients with consecutive powers of variable $\mathrm{x}$ allows for shaping the flight curve properly. The implemented guidance algorithm is based on a third degree polynomial 


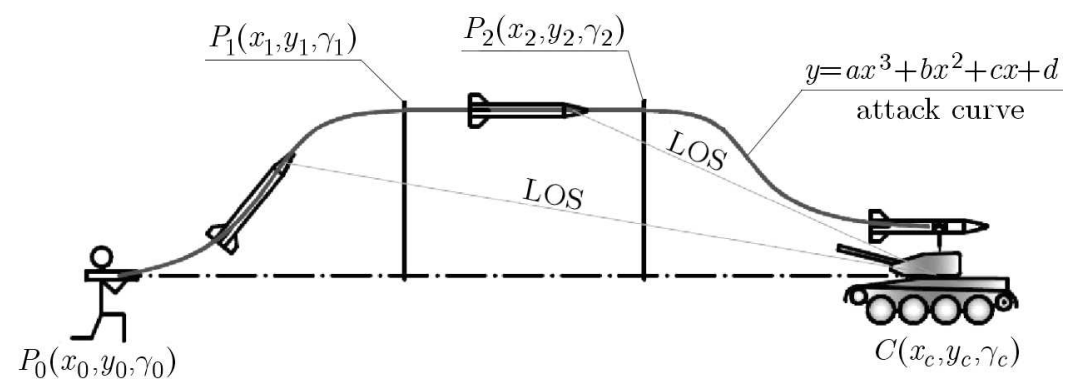

Fig. 4. Schematic diagram of ATGM attack along the line-of-sight (LOS) with the programmed flight set at a given height over the target in the vertical plane

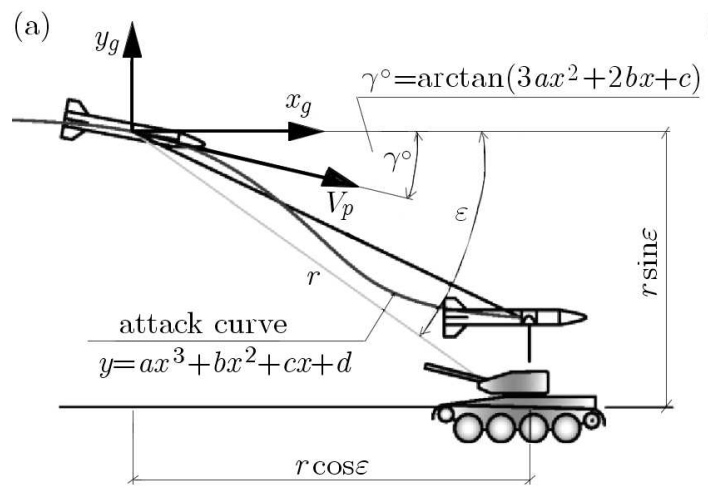

(b) fixing the position

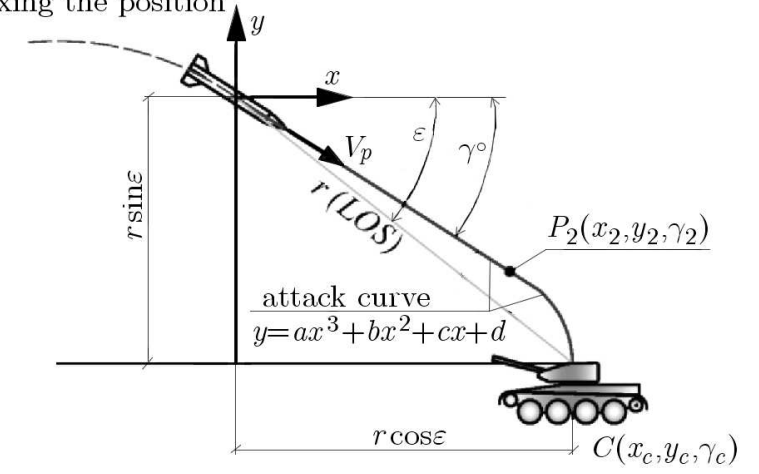

Fig. 5. View of the final stage of attack of ATGM flying on a polynomial curve; (a) missile flight above the target, (b) attack on the target from diving flight

curve. It gives the possibility of specifying not only the initial and final point of the flight, but also the angles of tangent inclination in those points. For determining the coefficients of the polynomial, it is necessary to know the coordinates of the initial point and the final point of the curve in the current flight phase. The missile flight trajectory is divided into consecutive phases with the beginning and end in given points. The coordinates of those two points comprise such coordinates as: location of a point on the vertical, horizontal axis and the axis of the tangent of inclination in that point

$$
\left(x_{0} ; y_{0} ; \gamma_{0}\right)\left(x_{k} ; y_{k} ; \gamma_{k}\right)
$$

From the set of four equations with four unknown values which are variable coefficients

$$
\begin{aligned}
& a x_{0}^{3}+b x_{0}^{2}+c x_{0}+d=y_{0} \\
& 3 a x_{0}^{2}+2 b x_{0}+c=\tan \gamma_{0} \\
& a x_{k}^{3}+b x_{k}^{2}+c x_{k}+d=y_{k} \\
& 3 a x_{k}^{2}+2 b x_{k}+c=\tan \gamma_{k}
\end{aligned}
$$

we get the following matrix equation to be solved

$$
\left[\begin{array}{cccc}
x_{0}^{3} & x_{0}^{2} & x_{0} & 1 \\
3 x_{0}^{2} & 2 x_{0} & 1 & 0 \\
x_{k}^{3} & x_{k}^{2} & x_{k} & 1 \\
3 x_{k}^{2} & 2 x_{k} & 1 & 0
\end{array}\right]\left[\begin{array}{l}
a \\
b \\
c \\
d
\end{array}\right]=\left[\begin{array}{c}
y_{0} \\
\tan \gamma_{0} \\
y_{k} \\
\tan \gamma_{k}
\end{array}\right]
$$

In the attack phase, when the target is moving, the polynomial coefficients are calculated at every moment of time as the final point of the curve is constantly changing its coordinates. Due 
to the limited computational power of the systems, the coefficients are presented in a cascading form (each consecutive coefficient includes the previous ones) (Grzyb and Koruba, 2011)

$$
\begin{aligned}
a & =-\frac{2 y_{k}-2 y_{0}+\left(x_{0}-x_{k}\right)\left(\tan \gamma_{k}+\tan \gamma_{0}\right)}{\left(x_{k}-x_{0}\right)^{3}} \\
b & =\frac{\tan \gamma_{0}-\tan \gamma_{k}-\left(3 x_{0}^{2}-3 x_{k}^{2}\right)}{2 x_{0}-2 x_{k}} \\
c & =\tan \gamma_{k}-3 x_{k}^{2} a-2 x_{k} b \quad d=y_{k}-x_{k}^{3} a-x_{k}^{2} b-x_{k} c
\end{aligned}
$$

We already know the curve the values of which correspond to the altitude of flight of ATGM at a given moment of time

$$
y=a x^{3}+b x^{2}+c x+d
$$

The angle of control $\gamma^{\circ}$ can be expressed with a formula

$$
\gamma^{\circ}=\arctan \left(3 a x^{2}+2 b x+c\right)
$$

In the initial flight phase, it is enough to use a proportional controller. The attack phase requires more accurate calculations. In the mentioned phase, the arguments of the PD controller are the tangent of the inclination angle to the path $\gamma$ and the flight altitude $y_{p}$

$$
Q=k_{1} e+k_{2} \frac{d e}{d t}+h_{1} f+h_{2} \frac{d f}{d t}
$$

where: $e=\gamma^{\circ}-\gamma, f=y-y_{p}, y_{p}$ - current ceiling of ATGM at a given moment of time, $y$ - altitude at which the ATGM should be at the given moment of time.

\section{Simplified equations of ATGM motion}

The algorithm determining the control force is implemented into a simplified mathematical model of missile motion in the vertical plane (Fig. 6) (Harris and Slegers, 2009; Koruba and Nocoń, 2012; Koruba and Osiecki, 2006).

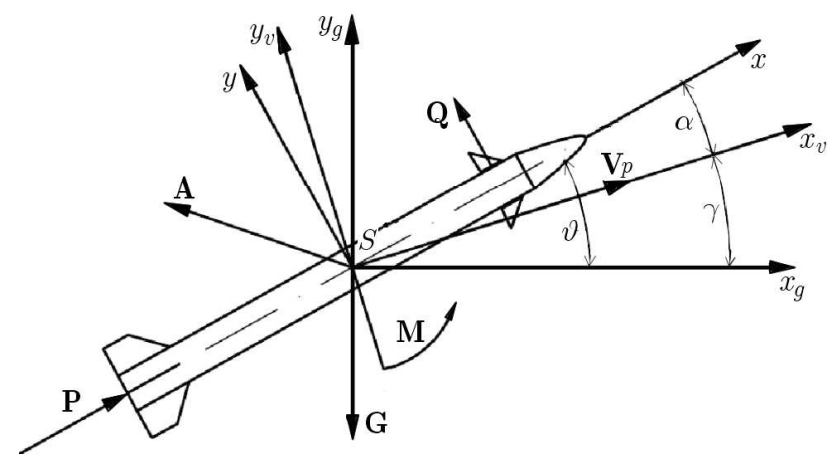

Fig. 6. The view of the rocket missile moving in the Earth's gravitational field and atmosphere, including the adopted systems of coordinates

The equations of flight dynamics of missile motion in the vertical plane in the system of coordinates connected with the flow $-S x_{v} y_{v} z_{v}$ have the following form (Harris and Slegers, 2009; Koruba and Nocon, 2012; Koruba and Osiecki, 2026)

$$
\begin{aligned}
& m \dot{V}_{p}=P \cos \alpha-G \sin \gamma-m \lambda_{x} V_{p}^{2} \\
& m V_{p} \dot{\gamma}=P \sin \alpha-G \cos \gamma+m \lambda_{y} \alpha V_{p}^{2}+Q \\
& \ddot{\vartheta}=-D_{1} \frac{V_{p}^{2}}{L} \alpha-D_{2} V_{p} \dot{\alpha}-D_{3} V_{p} \dot{\vartheta}+\frac{u Q}{J_{z}}
\end{aligned}
$$




\section{Obtained results}

Investigations have been conducted for a simplified hypothetical model of an anti-tank guided missile, both short- and long-range, attacking a target from the upper ceiling with flat and diving flight.

\subsection{Simulation results for short-range ATGM}

Figures 7 and 9 present the results of simulations conducted for a target $400 \mathrm{~m}$ away and moving with the velocity of $20 \mathrm{~m} / \mathrm{s}$.
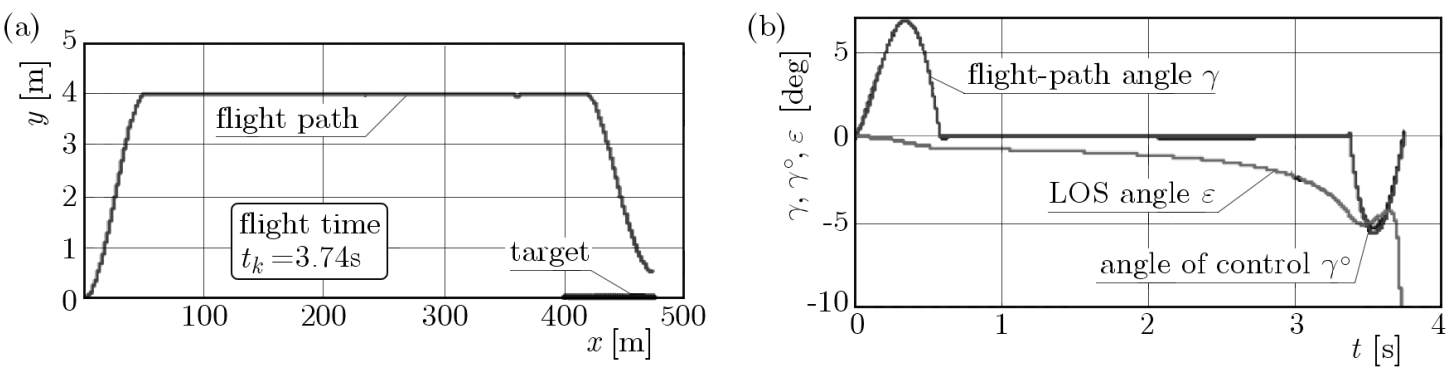

Fig. 7. (a) Flight path of ATGM heading towards a target $400 \mathrm{~m}$ away, (b) angles in function of time calculated during the flight

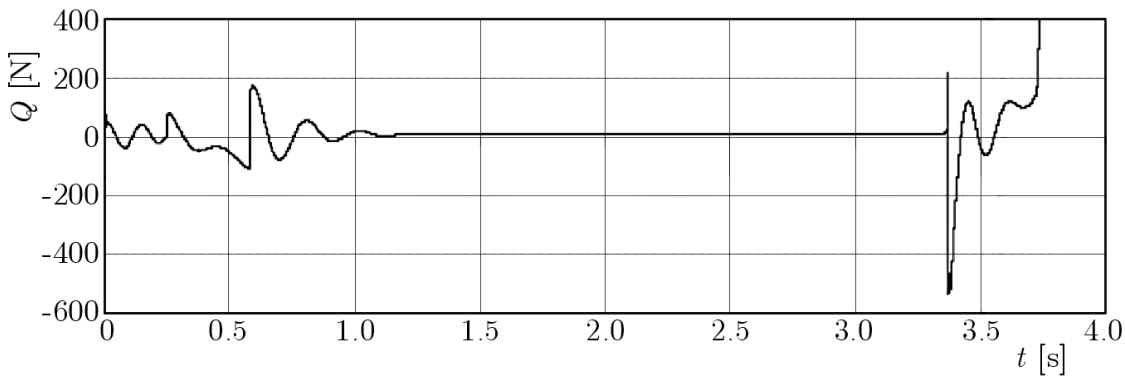

Fig. 8. Controlling force in function of time during the flight

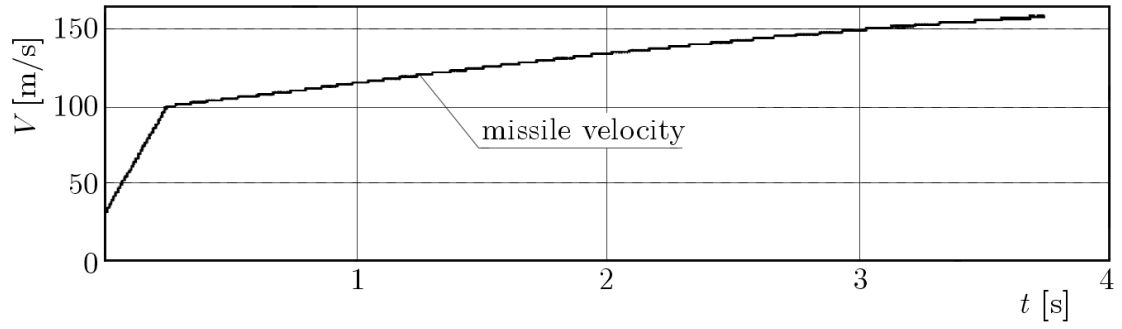

Fig. 9. ATGM velocity during the flight

Figures 10 and 12 present the results of simulations conducted for a target $600 \mathrm{~m}$ away. The target is moving with the velocity of $30 \mathrm{~m} / \mathrm{s}$ at an angle of $30^{\circ}$ (e.g. on a hillside).

\subsection{Simulation results for ATGM attacking a target from diving flight}

Figures 13 and 14 show the results of simulation conducted for a target $10 \mathrm{~km}$ away and moving with the velocity of $10 \mathrm{~m} / \mathrm{s}$.

Figure 15 and 16 present, in turn, the results of simulation conducted for a target $10 \mathrm{~km}$ away and moving with the velocity of $25 \mathrm{~m} / \mathrm{s}$ at an angle of $10^{\circ}$ (e.g. on a hillside). 


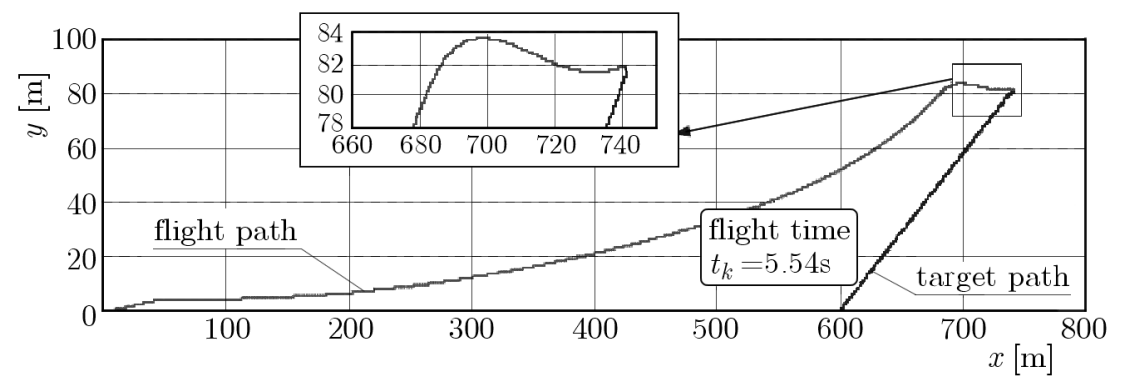

Fig. 10. Fights paths of ATGM and the target
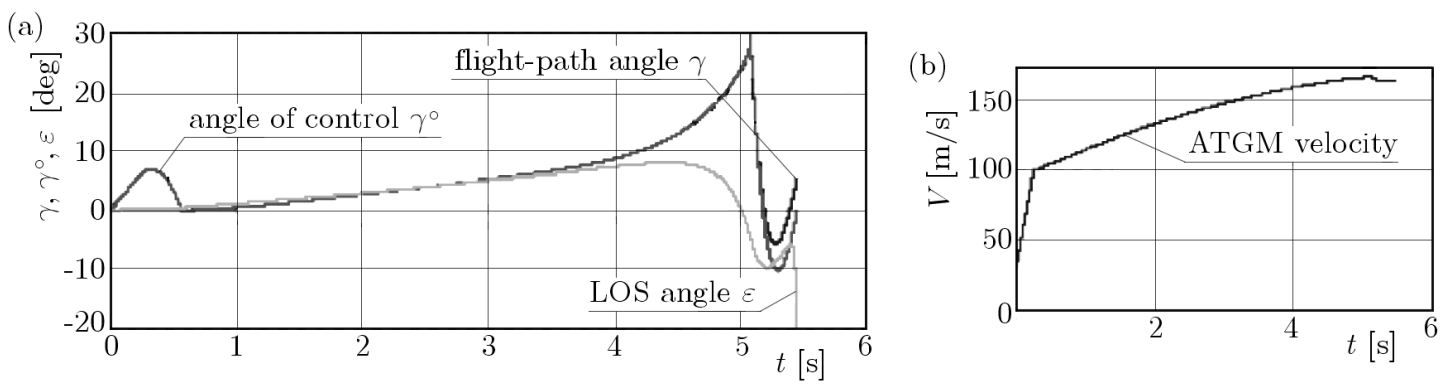

Fig. 11. (a) Angles in function of time during ATGM flight, (b) changes in velocity of ATGM in function of time

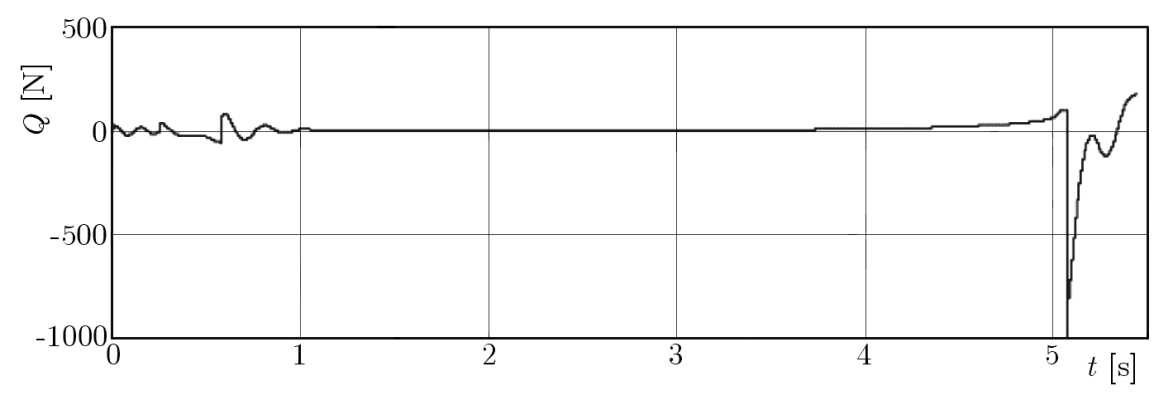

Fig. 12. Controlling force in function of time during the flight

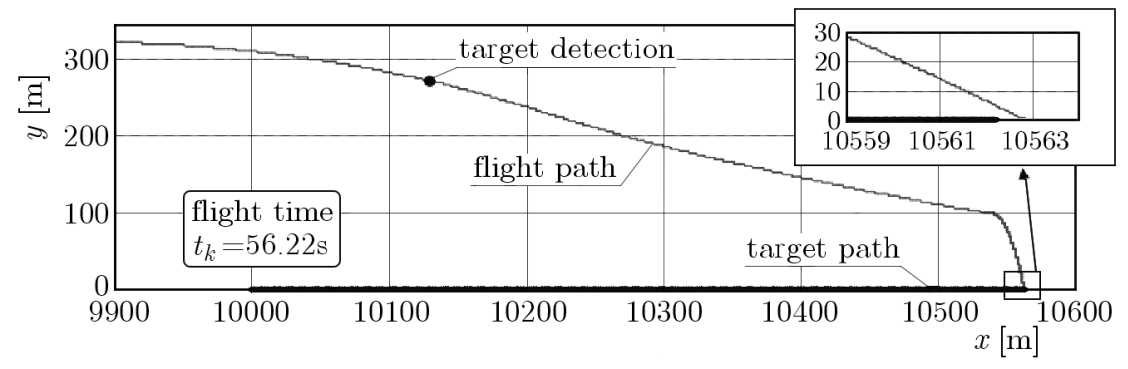

Fig. 13. The final stage of ATGM attack from diving flight moving towards the target 

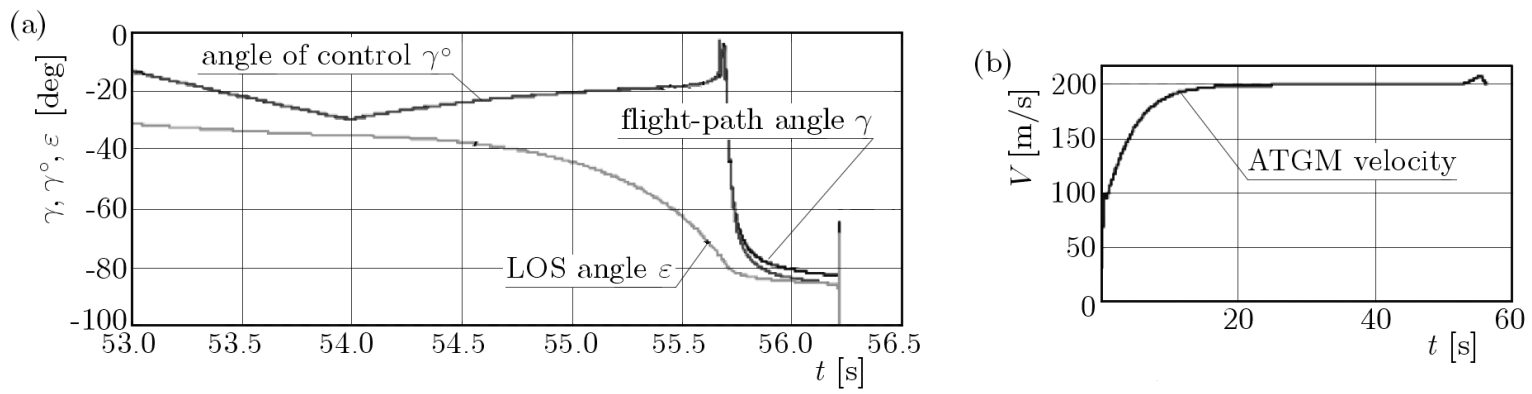

Fig. 14. (a) Angles of control, LOS and flight-path in function of time, (b) ATGM velocity during the flight
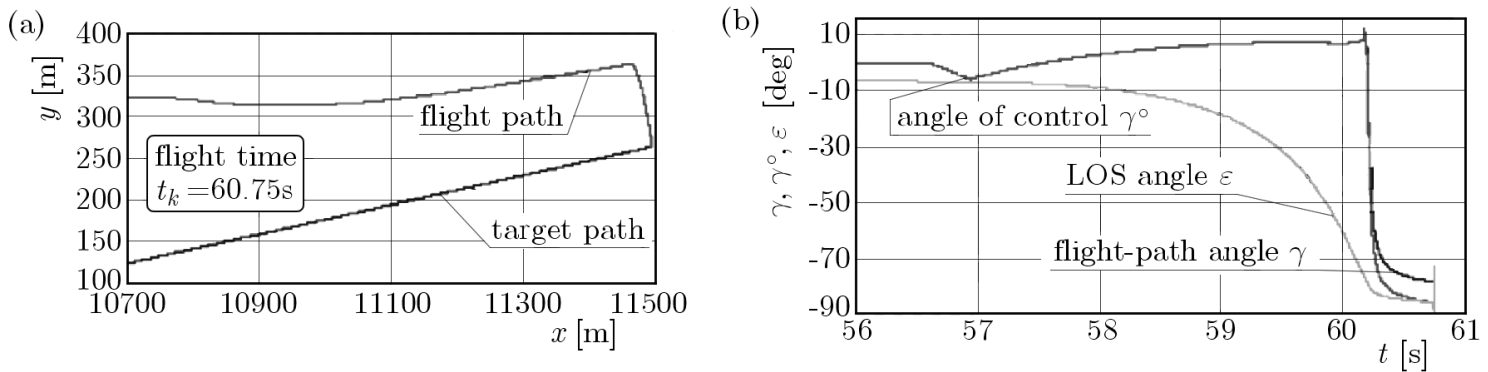

Fig. 15. (a) The final stage of attack from diving flight, (b) angle of control, LOS and flight-path in function of time

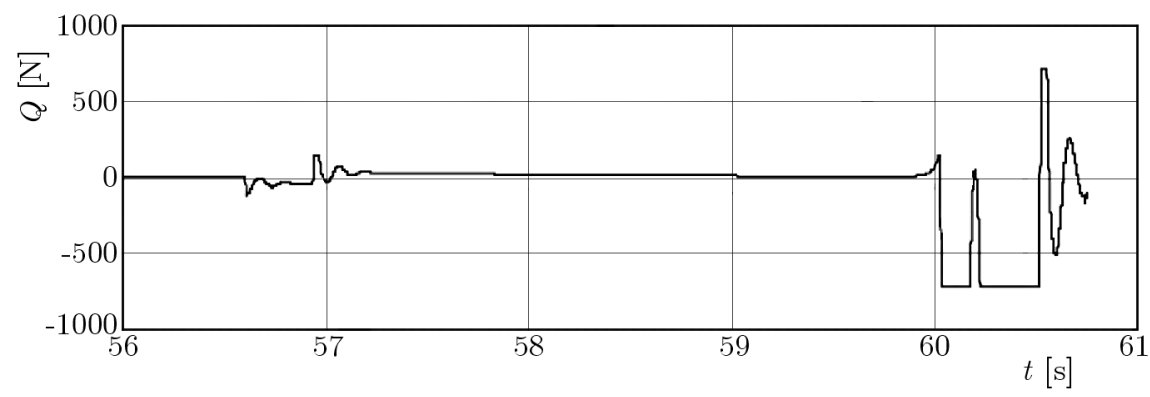

Fig. 16. Controlling force in function of time during the flight

\section{Conclusions and final remarks}

The following conclusions can be drawn from the theoretical considerations and simulation research:

- The simplified mathematical model of the missile moving only in the vertical plane is sufficient for the initial analysis of the guiding system of ATGM attacking a target from the upper ceiling. It allows verificationof the correctness of operation of the proposed algorithm.

- Effective short-range ATGM attack attacking a target along LOS occurs even in the case of exceeding, slightly, the real parameters of target motion (both the values as well as the direction of target motion).

- In the case of long-range ATGM attacking a target from diving flight, it can be seen that the accuracy of hitting is sufficient (within $0.5 \mathrm{~m}$ ).

- Long-range ATGM does not detect - hence does not hit - the target when it moves with 
the speed greater than $25 \mathrm{~m} / \mathrm{s}$ in the direction of the place of firing ATM. The target enters the locating area before the missile starts to search the set area.

- The angle of flight path during a dive attack is assumed to be $90^{\circ}$. However, due to trigonometrical limitations (non-uniqueness) which are applied to the algorithm, we adopt angles close to the assumed ones. An angle of $85^{\circ}$ ensures sufficient effectiveness of the attack.

Finally, it needs to be stated that the proposed algorithms of automatic control of an anti-tank guided missile work correctly during attack from the upper ceiling, both movable and fixed.

\section{References}

1. Evans D.M., 1990, The direct-fire anti-armour capability of UK land forces, Intern, Defense Review, 7, 739-742

2. Grzyb M., Koruba Z., 2011, Guiding a bomb to a sea target (in Polish), Zeszyty Naukowe Akademii Marynarki Wojennej, 185A, ISSN 0860-889X, 189-195

3. Harris J., Slegerrs N., 2009, Performance of a fire-and-forget anti-tank missile with a damaged wing, Mathematical and Computer Modelling, 50, 1/2, 292-305

4. Koruba Z., 2008, The Elements of the Theory and Applications of the Controlled Gyroscope (in Polish), Monographs, Studies, Dissertations M7, Kielce University of Technology, Kielce

5. Koruba Z., Nocoń Ł., 2012, Selected algorithms of automatic guidance of anti-tank rocket missiles attacking targets from upper ceiling (in Polish), Pomiary Automatyka Robotyka, 2

6. Koruba Z., Osiecki J., 2006, Structure, Dynamics and Navigation of Chosen Precision Kill Weapons, Publishing House of Kielce University of Technology, ISBN 83-88906-17-8, Kielce

7. Siouris G.M., 2004, Missile Guidance and Control Systems, Springer, New York

8. Yanushevsky R., 2008, Modern Missile Guidance, CRC Press Taylor \& Francis Group, New York

9. http://www.eng.uah.edu/ slegers/Publications/ 